\title{
Moth Flame Optimization Algorithm for Optimal FIR Filter Design
}

\author{
Zainab Muhammad Adamu \\ Department of Computer Engineering, Faculty of Engineering, University of Maiduguri, Maiduguri, Nigeria \\ E-mail: zainabmbarkindo@gmail.com
}

\section{Emmanuel Gbenga Dada}

Department of Mathematical Sciences, Faculty of Science, University of Maiduguri, Maiduguri, Nigeria

E-mail: gbengadada@unimaid.edu.ng

\author{
Stephen Bassi Joseph \\ Department of Computer Engineering, Faculty of Engineering, University of Maiduguri, Maiduguri, Nigeria \\ E-mail: sjbassi74@unimaid.edu.ng
}

Received: 10 July 2021; Accepted: 29 August 2021; Published: 08 October 2021

\begin{abstract}
This paper presents the application of Moth Flame optimization (MFO) algorithm to determine the best impulse response coefficients of FIR low pass, high pass, band pass and band stop filters. MFO was inspired by observing the navigation strategy of moths in nature called transverse orientation composed of three mathematical submodels. The performance of the proposed technique was compared to those of other well-known high performing optimization techniques like techniques like Particle Swarm Optimization (PSO), Novel Particle Swarm Optimization (NPSO), Improved Novel Particle Swarm Optimization (INPSO), Genetic Algorithm (GA), Parks and McClellan (PM) Algorithm. The performances of the MFO based designed optimized FIR filters have proved to be superior as compared to those obtained by PSO, NPSO, INPSO, GA, and PM Algorithm. Simulation results indicated that the maximum stop band ripples 0.057326, transition width 0.079 and fitness value 1.3682 obtained by MFO is better than that of PSO, NPSO, INPSO, GA, and PM Algorithms. The value of stop band ripples indicated the ripples or fluctuations obtained at the range which signals are attenuated is very low. The reduced value of transition width is the rate at which a signal changes from either stop band to pass band of a filter or vice versa is very good. Also, small fitness value in an indication that the values of the control variable of MFO are very near to its optimum solutions. The proposed design technique in this work generates excellent solution with high computational efficiency. This shows that MFO algorithm is an outstanding technique for FIR filter design.
\end{abstract}

Index Terms: Signal Processing, Finite Impulse Response (FIR), Moth Flame Optimization (MFO), Parks and McClellan (PM) Algorithm, Evolutionary Optimization.

\section{Introduction}

Digital filter is a set of procedure that can be exploited to proffer solution to mathematical computation executed in hardware and software that works on a digital output signal for the intent of attaining a filtering objective [1]. Digital Filters are widely used in digital signal processing, as its area of application include digital communication system, biomedical signal processing, electronics and computer music [2]. Digital signal processing is an important and growing subject area in computer engineering, as FIR is a filter configuration that can be employed to execute virtually all kind digital frequency response. FIR filter has the ability of attenuating some frequencies, amplifies others and emit out an output of finite length [3].

Digital filters are classified into two: Finite Impulse Response (FIR) and Infinite Impulse Response (IIR) digital filters. Finite Impulse Response (FIR) filter is one of the major types of digital filter which is defined as a filter whose response to any finite length input is of finite duration and it has no feedback which makes it always stable [4]. It can be exploited to realize nearly every type of digital frequency response and is specific to sample system. Whereas Infinite Impulse Response (IIR) filters is a filter with infinite impulse response which has feedback from output to input and that makes the transfer function more complicated [4]. FIR a finite-impulse-response filter with linear stage that guarantees that all frequency signals are slowed by the same time period, in so doing eradicating the likelihood of stage deformation while IIR have non-linear phase that is difficult to control which makes it unstable [5]. All filters are repetitive surface intended to replicate, convey or take in electromagnetic fields depending on the frequency. They are 
normally utilized to isolate diverse frequencies from some group of substantial frequencies in relation to their parameter that is referred to as the cutoff frequency [6]. These are low pass, high pass, band pass and band stop filters. The general difference equation for the FIR filters is

$$
y(n)=\sum_{k=0}^{n-1} b_{k} x(n-k)
$$

Given that $y(n)$ is the filter output at discrete time instance $n, b_{k}$ is the $k$-th feed forward tap, or filter coefficient, and $\mathrm{x}(\mathrm{nk})$ is the filter input lagged by $\mathrm{k}$ samples. The $\sum$ depicts the sum from $\mathrm{k}=0$ to $\mathrm{k}=\mathrm{n}-1$ where $\mathrm{n}$ is the number of feed forward taps in the FIR filter or filter length. Finite Impulse Response (FIR) filter transfer function is presented as below:

$$
H(z)=\sum_{k=0}^{N} h(n) z^{-k}
$$

Assuming that $\mathrm{N}$ is the order of the filter and $\mathrm{h}(\mathrm{n})$ as the impulse response of the filter.

Researchers and engineers have applied different techniques to design FIR filters. These techniques have demonstrated that they are very effective by delivering fast convergence and high performance. However, there is still a need to improve and overcome some of the challenges associated with existing filters that makes such filters to converge very fast with ripples but sometimes gets trap in local minima, and has low band pass and stop band ripples. Moreover, techniques such as the one proposed by [7] that used Blackman and Flat Top Window method has slow convergence and high ripples. Another downside of the existing filters lower ripples and fitness values such as the FIR filters using improved PSO designed by [8]. To address these challenges, the Moth Flame Optimization (MFO) algorithm which is presented in this paper. MFO has the capacity to converge fully, and obtaining global optimum. It also improves the robustness and accuracy of system which provides minimum fitness value, pass band and stop band ripples that is magnitude response [9].

Softcomputing techniques has become popular for solving signal processing problems because of their ease of implementation and fewer tuning parameters that are needed to make it attain the global optimal. Several bio-inspired algorithms have been used for optimisation of digital filters. Particle swarm optimization (PSO) [4, 10, 14, 15, 16], Genetic Algorithm (GA) [17, 18], Fuzzy Logic [19], and Adaptive neuro-fuzzy inference system (ANFIS) [20]. Hybrid techniques such as Simulated annealing and PSO [21], Artificial Neural Network (ANN), Genetic Algorithms and Fuzzy Set [22], Cat Swarm Optimisation (CSO) and Fast Library for Approximate Nearest Neighbours (FLANN) [23], and the combination of GA, NN, and Fuzzy Logic [24].

The MFO algorithm has become popular over the years and it is been used in solving analogous problems in various fields. The research tends to employ Moth Flame Optimization algorithm which has the capability to select the best solution and provides the global optimum transition width, reduces the error and improves its performance. The most interesting fact about moths is their special navigation methods in the night; they have been evolved to fly at night using the moon light. They utilize a mechanism called transverse orientation for navigation. In this method, a moth flies by maintaining a fixed angle with respect to the moon, a very effective mechanism introduced by Mirjalili and tested on 29 benchmark functions and seven real engineering problems. It was also compared with several algorithms which include PSO, GA, and ACO which have been experimentally proved that it has better performance, low-cost design, accuracy and good robustness. MFO is of immense advantage over others. This includes: MFO has high convergence accuracy and good global optimization ability in optimization problem for week dimensional function, which is superior to PSO algorithm. Accuracy of MFO algorithm is higher than PSO algorithm. MFO algorithm has good robustness, fast convergence speed and global optimization. MFO algorithm is an effective technique to estimate the parameters of confined aquifer, and also effectively conducts parameter inversion for underground water model.

It was discovered after reviewing relevant literature that there is the non-existence of work on digital filter design using MFO. Moreover, many of the existing works on digital filter design have low performance, while some did not use state-of-the-art metrics to evaluate the performance of their work. Furthermore, some authors did not compare their work with high performing Softcomputing algorithms. Bearing in mind such inadequacy, the novelty of this work centers on the use of the design of digital filter using MFO and an intensive comparison of different Softcomputing algorithms used for FIR filter design. The aim of this paper is to design an optimized FIR filters using Moth Flame Algorithm (MFO). The specific objectives of the paper are:

- To obtain the filter coefficient of the FIR filters using MATLAB

- To evaluate and determine the magnitude response (pass band, stop band ripples and fitness value) using the MFO algorithm

- To evaluate performance of the system using standard performance metrics.

- To compare the performance of the system with state of the algorithms mentioned in Khalid and Nemer [8].

The contribution of this work is summarised as follows: 
- An up-to-date brief survey of Softcomputing techniques that have been applied to digital filter design is presented.

- An MFO algorithm that overcomes the shortcomings associated with the existing techniques that have been applied for the design and optimisation of low pass digital filters is proposed.

- A comparative analysis of high-performance soft computing algorithms used for FIR filter designs.

\section{Related Work}

Several researches have been conducted during the past years to design filters. The most recent research is using several types of optimization techniques, which include Particle Swarm Optimization (PSO) used by Vijay and Venkata [10] to determine the frequency response of digital FIR low pass filters through random algorithm which find the filter coefficient. Rakhi and Kavita [11] described FPGA implementation of FIR filter for DSP application such that the paper proposed a multiplier less method, founded on add and shift technique. It performs at a rate that significantly surpass those of the state-of-the-art programmable DSP. The Field Programmable Gate Array (FPGA) is hardware-based technique and therefore makes it more time consuming, it also lacks the ability to provide the optimization features.

Kaur and Dhaliwal [12] designed low pass filter using Artificial Neural Network (ANN) considering feed forward neural network with radial basis function for training it. Uthayakumar and Rabi [13] considered a FIR filter using rectangular window method, which analyze its performance for various frequency responses. Simulations were carried out using MATLAB for the FIR filter with order 4, order 8, order 12 and order 18 for low pass filter, high pass filter, band pass filter and band stop filters. Window method is a traditional method which needs to be optimizing by obtaining the impulse response and the rectangular window method was limited to four different order which might have more probability to error function.

Maan et al [14] worked on high order Digital Infinite Impulse Response (IIR) filter using Particle Swarm Optimization. PSO was used to evade being trapped in the local minima, improve the search ability and offers quick convergence for computing the optimal filter coefficients. PSO algorithm sometimes get trap at local minima, and also research shows MFO has better performance and obtains global optimum.

Jatana and Sidhu [4] worked on the design of Digital FIR High pass filter employing PSO which has the ability to converge to the required optimal result with limited number of iterations. Khalid and Nemer [8] performed an optimal design of linear phase filters considering high pass, low pass, band pass and band stop using a novel version of Particle Swarm Optimization (NPSO) technique. Therefore, as the need of filters is in wide range of application, it is of important to further minimize error, increase it filter order in order to avoid premature convergence and been trapped in the local minimal. As MFO has the capability to select the best solution and provides the global optimum transition width. This research intends to use Moth Flame Optimization (MFO) algorithm to design a Finite Impulse Response (FIR) filter.

\section{Methods}

\subsection{MFO Algorithm}

The Moth Flame Optimization (MFO) algorithm is a new evolution optimization algorithm based on moths' nighttime movement mechanism of having a constant alignment with the moonlight. The Moth Flame Optimization algorithm process for updating positions enables the generation of nearby solutions around the flames, which is primarily used to stimulate exploitation. Allocating a flame to every other moth boost search space exploration and reduces the likelihood of local optima stalling. Because MFO uses a population to address real-world problems using unexpected and confined moth search areas, local optima evasion is strong. Since moths usually try to update their positions in relation to flames, which are the most probable solutions gotten thus far throughout the period of iterations, the MFO algorithm's optimization and convergence are assured.

MFO algorithm has become popular over the years and it is been used in solving analogous problems in various field. This work employs MFO algorithm which has the capability to select the best solution and provides the global optimum transition width, reduce the error and improve its performance. The greatest fascinating aspect of moths is their unique nighttime navigation capabilities; they have adapted to travel at night utilizing moonlight [25]. They navigated using a technique known as transverse orientation. A moth travels by keeping a stationary angle with respect to the moon in this technique, which was developed by Mirjalili [25] and validated on 29 benchmark functions and seven real-world engineering challenges. It was also compared with several algorithms which include PSO, GA, ACO and others. It has been proved that it has better performance, low-cost design, accurate and good robustness.

\subsection{Proposed FIR Filter Design}

FIR filter is designed using MATLAB considering low pass, high pass, band pass and band stop filters. The magnitude response and normalized magnitude response of FIR filters were obtained considering the pass band normalized cut off frequencies and stop band normalized cut off frequencies given: 
$w_{p}=0.45, w_{s}=0.55$, ind $_{p}=\left(\frac{w}{p_{i}}<=w_{-} p\right)$ and ind $_{p}=\left(\frac{w}{p_{i}}>=w_{-} p\right)$.

Basic Steps of MFO Algorithm for Optimization of Low Pass FIR Filter

Step 1: Set initial value for the parameters Search Agents number/Number of Moths = 50, maximum number of flame position, $\mathrm{N}_{\text {fmax }}=50$, maximum number of iteration $\mathrm{T}=200$, minimum and maximum number of filter coefficients lower bound $\mathrm{lb}=-1$, upper bound $\mathrm{up}=1$, filter order/number of flame position $\mathrm{N}=20$, number of filter coefficient $\mathrm{h}(\mathrm{n})$.

Step 2: Generate the initial position of moth randomly in search space and calculate the objective function

$$
M(i, j)=(u b(i)-l b(i) * \operatorname{rand}+l b(i))
$$

Where $\mathrm{M}(\mathrm{i}, \mathrm{j})$ is the position of the moth while ub and lb defines the upper and lower bounds of the variables.

Step 3: Compute the fitness function, tag the best position of flame and bring up-to-date the position of moth regarding flame $M_{i=} S\left(M_{i}, F_{j}\right)$

Step 4: Bring up to date the number of flames, f using the equation

$$
\text { flame no }=\text { round }\left(N_{f}-l * \frac{N_{f}-1}{T}\right)
$$

Where $\mathrm{N}_{\mathrm{f}}$ is the maximum number flames, 1 is the current number of iterations and $\mathrm{T}$ is the maximum number of iterations.

Step 5: Compute D for the matching moth

$$
D_{i}=\left|F_{j}-M_{i}\right|
$$

Where $D_{i}$ specifies the distance of the $i$-th moth for the $j$-th flame, $M_{i}$ designates the $i$-th moth and $F_{j}$ specifies the $\mathrm{j}$-th flame.

Step 6: Bring up to date $\mathrm{M}_{\mathrm{i}, \mathrm{j}}$ for the corresponding moth

Step 7: If it attains the required number of simulations at maximum iterations report the best position among the moths and stops else go to step 3

\section{Results and Discussion}

This section presents the results of our simulations. The experimental setup and parameter setting of the MFO algorithm is described here. The impact of haphazard initialization, fifty trails were performed for each algorithm considered in this work and the maximum stop band ripples, transition width and fitness value values of objective function have been computed.

\subsection{Experimental Setup and Parameter Settings}

Some parameters were utilized to get the best filter coefficients of the filters while the magnitude response and normalized magnitude response of the low pass FIR filter graph were obtained considering the pass band ripples, stop band ripples, pass band normalized cut off frequencies and stop band normalized cut off frequencies. Different optimization test function was used to assess the performance of the MFO, and the result is compared with the ones obtained from previous filter optimization methods. The simulation was done using MATLAB and the parameter setting for the experiments conducted are as represented in Table 1 below:

Table 1. MFO Optimization parameters

\begin{tabular}{|c|c|}
\hline Parameters & Values \\
\hline Search Agents Numbers/Number of Moths & 50 \\
\hline Maximum Iteration, T & 200 \\
\hline Filter Order/ No. of flame position, N & 20 \\
\hline Upper bound (ub) & 1 \\
\hline Lower bound (lb) & -1 \\
\hline Number of Simulation & 50 \\
\hline
\end{tabular}

These parameters were used to design for low pass, high pass, band pass and band stop filters and obtained different results. 


\subsection{Simulation Results and Discussion}

This section presents the results from the experiments carried out using MATLAB 2018 software. From the experiments conducted, LP, HP, BP and BS FIR filters of the order of 20 have been designed for optimum performance. The total population for each algorithm was set at 50 with 200 iterations. After a number of trials, the algorithm's parameters were determined. To investigate the impact of random selection, fifty trials were run for each algorithm in this work, and the maximum stop band ripples, transition width, and fitness value values of the objective function were calculated. The optimal filter coefficients for FIR of order 20 are presented in Table 2.

Table 2. Optimized filter coefficients for FIR of order 20

\begin{tabular}{|c|c|c|c|c|}
\hline Filter Order & Low Pass & High Pass & Band Pass & Band Stop \\
\hline $\mathrm{H}(1)=\mathrm{h}(21)$ & 0.008243871400286 & -0.003424720342443 & -0.030996834931252 & 0.015014303804295 \\
\hline $\mathrm{H}(2)=\mathrm{h}(20)$ & 0.014994715535941 & 0.018327403184361 & 0.000072528611111 & -0.056505586108589 \\
\hline $\mathrm{H}(3)=\mathrm{h}(19)$ & -0.017443754600866 & 0.008604476791348 & 0.077071194087740 & 0.019122466265923 \\
\hline $\mathrm{H}(4)=\mathrm{h}(18)$ & -0.038404247724173 & -0.039557147088955 & -0.000156644474290 & -0.012714332140646 \\
\hline $\mathrm{H}(5)=\mathrm{h}(17)$ & 0.016510490784612 & -0.002704229036757 & -0.046775375499441 & 0.004719706975430 \\
\hline $\mathrm{H}(6)=\mathrm{h}(16)$ & 0.067558080362764 & 0.068441127986157 & 0.000240246536519 & 0.004719706975430 \\
\hline $\mathrm{H}(7)=\mathrm{h}(15)$ & -0.001362869095272 & -0.011006311951809 & -0.098358616726847 & -0.012223415435265 \\
\hline $\mathrm{H}(8)=\mathrm{h}(14)$ & -0.112671125883860 & -0.109763005197377 & -0.000234074260575 & -0.082576203715115 \\
\hline $\mathrm{H}(9)=\mathrm{h}(13)$ & -0.012871453147150 & 0.025553492845641 & 0.295822817967064 & -0.015419007843788 \\
\hline $\mathrm{H}(10)=\mathrm{h}(12)$ & 0.292938773142360 & 0.293017677863764 & 0.000089144174071 & -0.527277895972626 \\
\hline $\mathrm{H}(11)$ & 0.471744840981952 & -0.481959324767428 & -0.384646132704533 & -0.012047788089896 \\
\hline
\end{tabular}

The optimized LP filter coefficients obtained by Particle Swarm Optimization (PSO), Novel Particle Swarm Optimization (NPSO), Improved Novel Particle Swarm Optimization (INPSO), Genetic Algorithm (GA), Parks and McClellan (PM) Algorithm and Moth Flame Optimization (MFO) algorithm is presented in Table 3. It is obvious that the maximum stop band ripples of 0.057326 obtained by MFO is better than that of PSO, NPSO, INPSO, GA, and PM Algorithm. The experimental results also validate the superior search capability of MFO when compared to other optimization methods used in this paper. The second performance parameter named 'transition width' is genuinely pleased by all methods. With the intention to ascertain the computational performance, the fitness value is introduced as the third performance parameter, MFO having the best fitness value of 1.3682 indicated that the algorithm have the capability to generate enhanced solution in comparison to other soft computing algorithms used in this study. Fitness value determines the constant pass band and stop band ripples, therefore the lower the fitness value the lower the ripples in both bands.

Table 3. Comparative results of performance parameters for LP filter order 20.

\begin{tabular}{|c|c|c|c|}
\hline Algorithms & Maximum stop band ripples & Transition Width & Fitness value \\
\hline PSO & 0.02966 & 0.0802 & 2.357 \\
\hline NPSO & 0.026411 & 0.077 & 4.4587 \\
\hline INPSO & 0.021601 & 0.075 & 4.1673 \\
\hline GA & 0.055583 & 0.080 & 3.6851 \\
\hline PM & 0.057154 & 0.0803 & 4.5499 \\
\hline MFO & 0.057326 & 0.079 & 1.3682 \\
\hline
\end{tabular}

Depicted in Table 4 is the optimized HP filter coefficients obtained by PSO, NPSO, INPSO, GA, PM and the proposed MFO method. It is evident that the maximum stop band ripples of 0.057326 obtained by MFO is better than that of PSO, NPSO, INPSO, GA, and PM Algorithm.

Table 4. Comparative results of performance parameters for HP filter order 20

\begin{tabular}{|c|c|c|c|}
\hline Algorithm & Maximum stop band ripple & Transition width & Fitness value \\
\hline PSO & 0.040289 & 0.0806 & 2.225 \\
\hline NPSO & 0.068797 & 0.080 & 2.2964 \\
\hline INPSO & 0.12103 & 0.085 & 3.5905 \\
\hline GA & 0.05409 & 0.081 & 3.77 \\
\hline PM & 0.057154 & 0.0804 & 4.5729 \\
\hline MFO & 0.11946 & 0.079 & 1.5492 \\
\hline
\end{tabular}

Presented in Tables 5 and 6 are the comparative results of performance parameters for BP and BS filters. 
Table 5. Comparative results of performance parameters for BP filter order 20

\begin{tabular}{|c|c|c|}
\hline Algorithm & Maximum stop band ripples & Fitness value \\
\hline PSO & 0.040196 & 2.0667 \\
\hline NPSO & 0.02173 & 2.7917 \\
\hline INPSO & 0.034811 & 2.7686 \\
\hline GA & 0.063713 & 2.4178 \\
\hline PM & 0.061886 & 4.0877 \\
\hline MFO & 0.033788 & 1.6811 \\
\hline
\end{tabular}

Table 6. Comparative results of performance parameters for BS filter order 20

\begin{tabular}{|c|c|c|}
\hline Algorithm & Maximum stop band ripples & Fitness value \\
\hline PSO & 0.058494 & 3.9799 \\
\hline NPSO & 0.026186 & 3.4522 \\
\hline INPSO & 0.015899 & 3.8272 \\
\hline GA & 0.087204 & 5.2516 \\
\hline
\end{tabular}

The $\mathrm{dB}$ and normalized plot for LP, HP, BP and BS FIR filters are presented in Figs. 1 - 8 respectively.

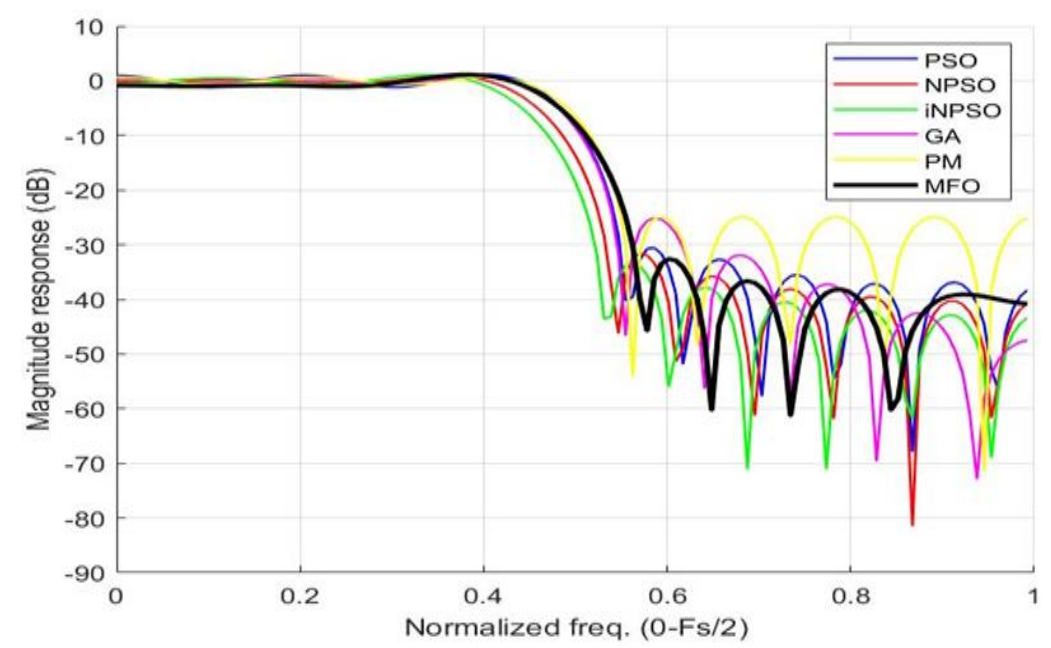

Fig.1. dp plot low pass filter

Fig. 1 depicts the magnitude response in decibel $(\mathrm{dB})$ of a low pass filter which shows a maximal reduction of ripples in the pass band, as they are fluctuations that affects signal flow, as such the lower the ripples in the pass band of the filter the better the signals to be obtained. Minimal ripples indicate the presence of fewer distortions, as it also has a higher transition before attenuating the signals to its stop band. The ripples in the stop band shows presence of fluctuations where already the signal has been attenuated therefore has no effect on any signal, and also the magnitude can have small ripples never be constant only for an ideal filter.

Fig. 2 indicates normalized magnitude response of a low pass filter which shows a maximal reduction of ripples in the pass band, as they are fluctuations that affects signal flow, as such the lower the ripples in the pass band of the filter the better the signals to be obtained. Minimal ripples indicate the presence of fewer distortions, as it also has a higher transition before attenuating the signals to its stop band. The ripples in the stop band are also minimal has been attenuated therefore has no effect on any signal.

From Fig. 3, the magnitude response $(\mathrm{dB})$ graph for high pass filter. It shows that the maximum stop band ripples in the stop band which indicates fluctuations and signals are attenuated in the range, while maintaining a high transition from the stop band to pass band. The $\mathrm{dB}$ plot means been measured in decibels within the range of (10 to -90$) \mathrm{dB}$. The pass band is the rate at which the filter allows signal to pass through, which from our graph above has less ripples therefore minimizing distortions or fluctuations in a signal. 


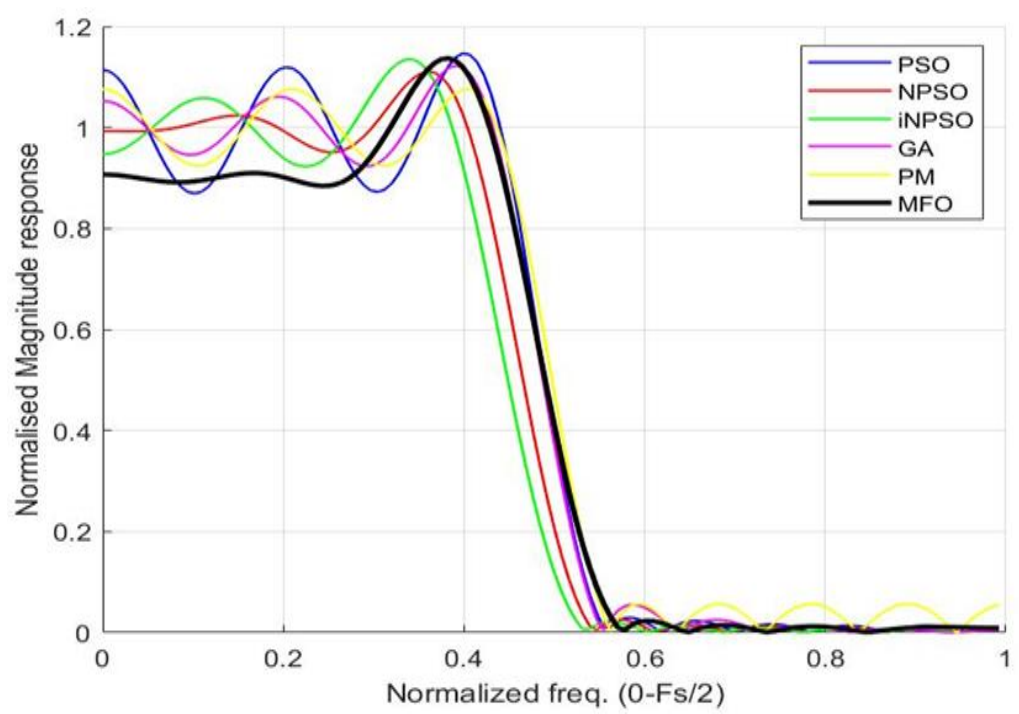

Fig.2. Normalised plot low pass filter

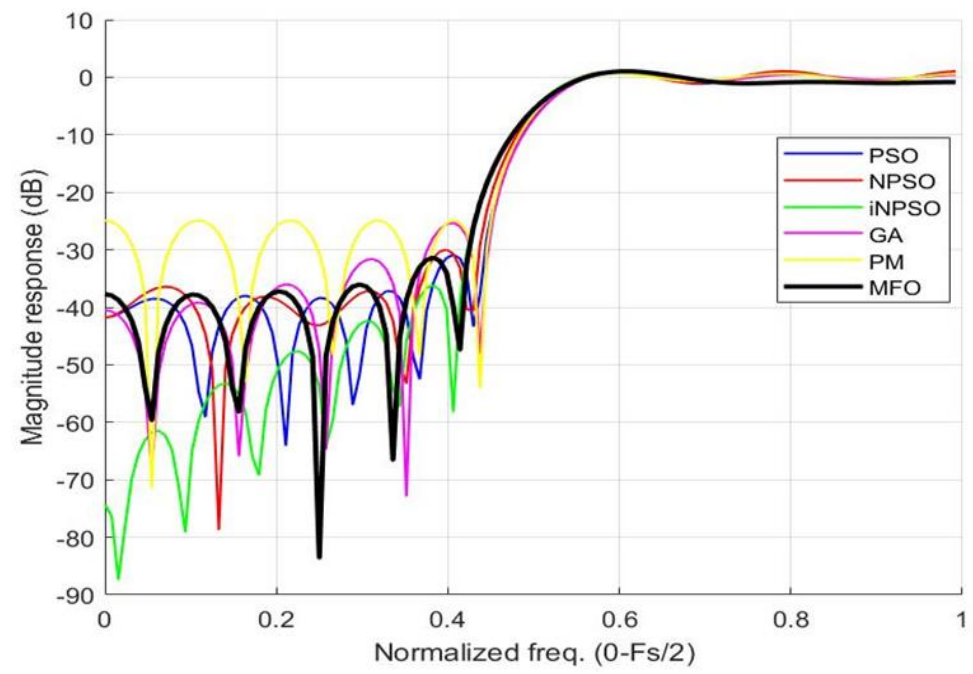

Fig.3. Dp plot high pass filter

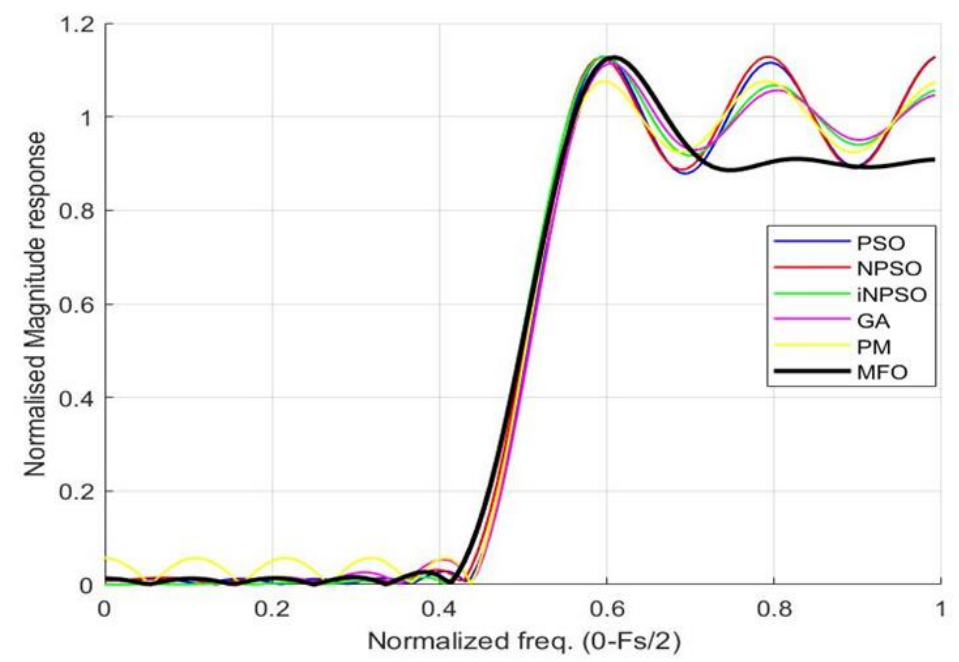

Fig.4. Normalised plot high pass filter

Depicted in Fig. 4 is the normalized magnitude response graph for high pass filter. It shows maximum stop band ripples which indicates no signal passing within the range which maintains a high transition from the stop band to pass band. The pass band is the rate at which the filter allows signal to pass through, which from our graph above has less 
ripples therefore minimizing distortions or fluctuations in the signal. The normalized plot we rescale it to an average of (0 to 1.5$) \mathrm{Hz}$ for all the filters.

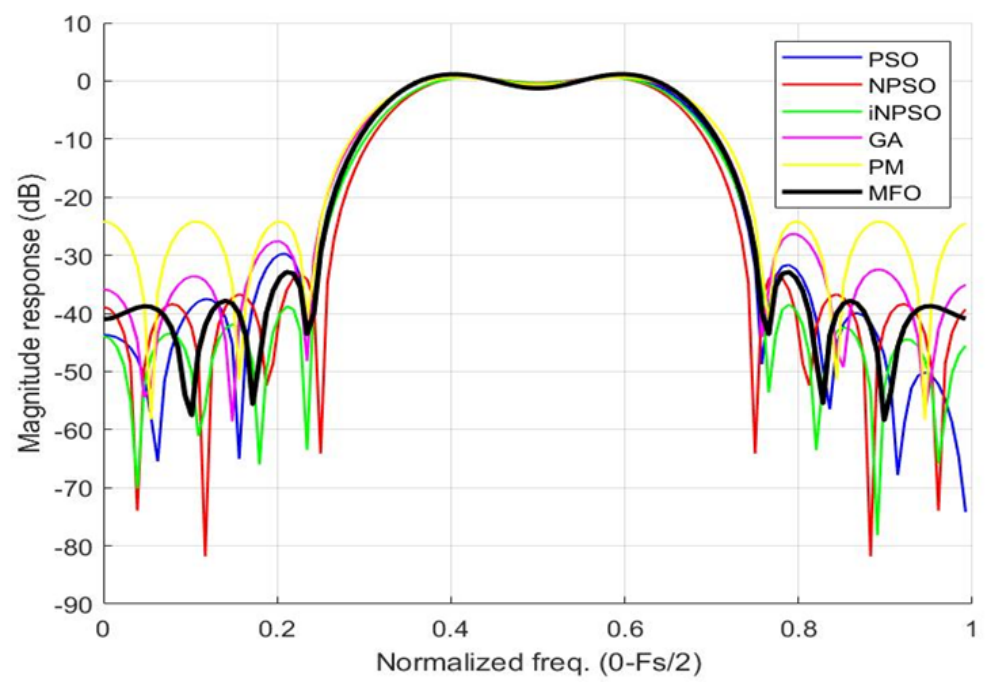

Fig.5. Dp plot Band pass filter

Fig. 5 represents the magnitude response $(\mathrm{dB})$ graph for band pass filter. It shows maximum stop band ripples within its stop band, which indicates presence of fluctuations as signal is been attenuated maintains a high transition from the stop band to pass band. The pass band is the rate at which the filter allows signal to pass through, which from our graph above has less ripples therefore minimizing distortions or fluctuations in the signal.

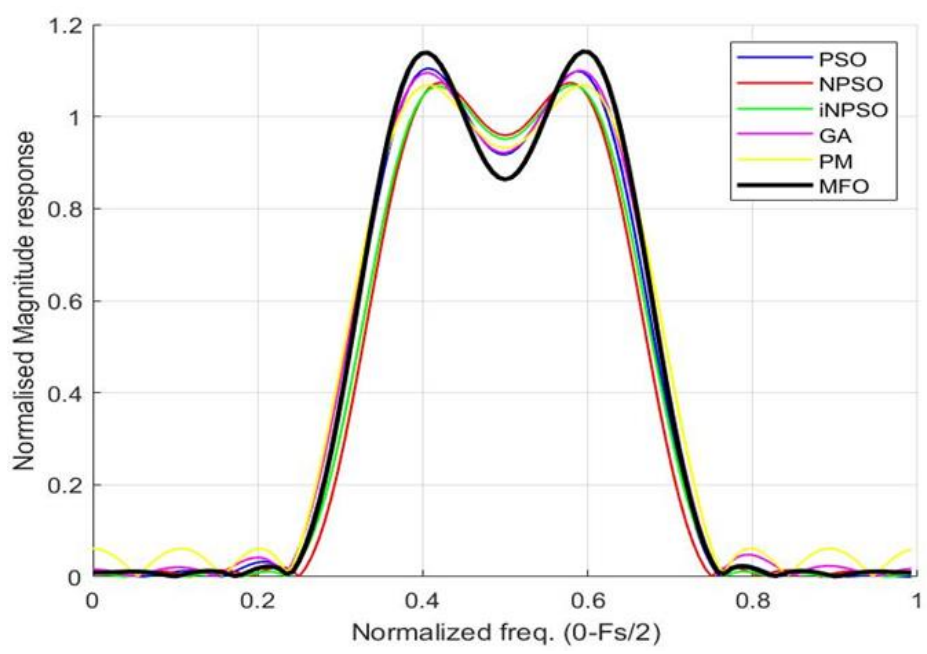

Fig.6. Normalised plot Band pass filter

Fig. 6 illustrates normalized magnitude response of a band pass filter identifying its pass band and stop band of the FIR filters. The stop band which indicates the rate at which the signal is been attenuated in the graph from 0.38 to 0.62 has more ripples than the pass band. The pass band shows a minimal ripple while maintaining a transition width before reaching its attenuated stage which is the stop band.

Fig. 7 illustrates magnitude response $(\mathrm{dB})$ of a band stop filter identifying its pass band and stop band of the FIR filters. The stop band which indicates the rate at which the signal is been attenuated in the graph from 0.38 to 0.62 has more ripples than the pass band. The pass band shows a minimal ripple while maintaining a maximal transition before reaching its attenuated stage which is the stop band.

Fig. 8 above illustrates a normalized magnitude response of a band stop filter identifying its pass band and stop band of the FIR filters. The stop band which indicates the rate at which the signal is been attenuated in the graph from 0.38 to 0.62 has more ripples than the pass band. The pass band shows a minimal ripple while maintaining a maximal transition before reaching its attenuated stage which is the stop band. 


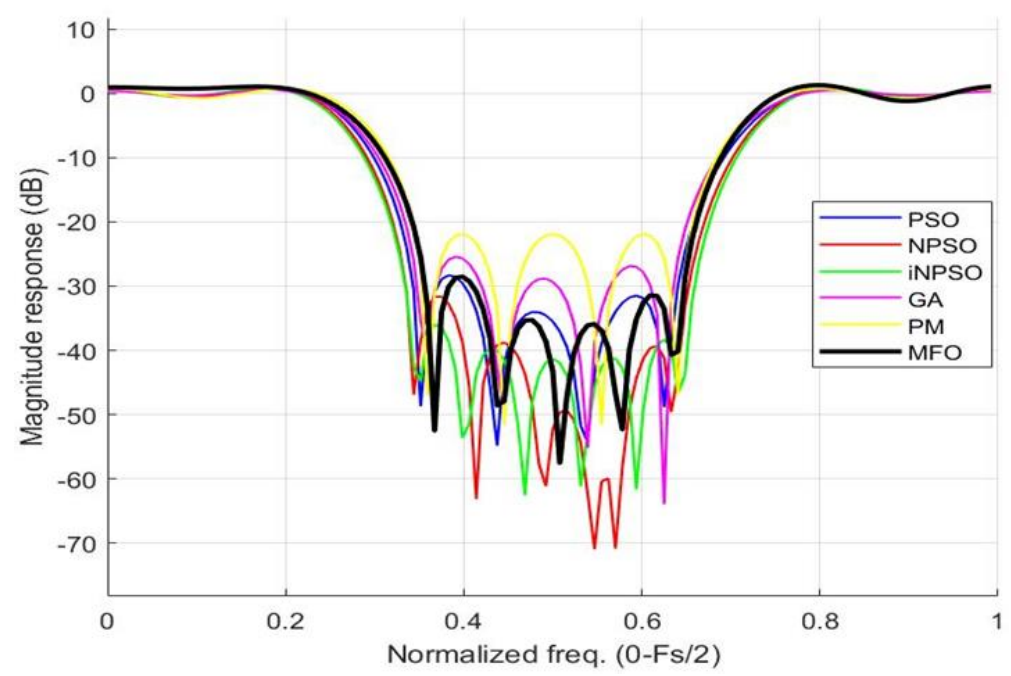

Fig.7. Dp plot Band stop filter

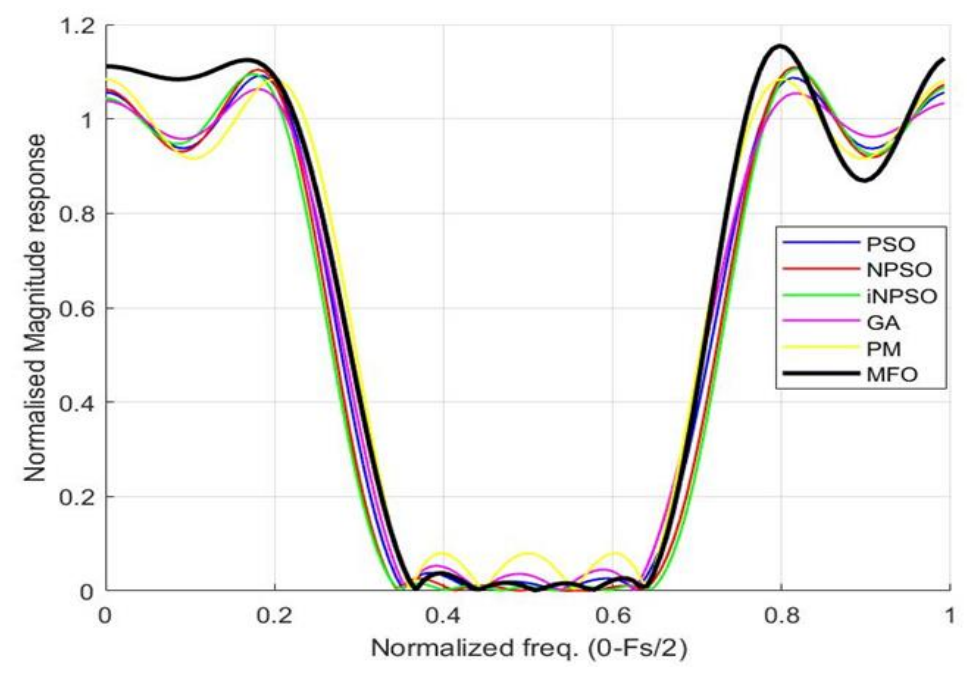

Fig.8. Normalised plot Band stop FIR filters

\section{Conclusions}

This paper presents an optimally designed FIR filter using MFO. The proposed method exploits the strengths of MFO algorithm. The proposed approach has been utilized for an optimum design of LP, HP, BP and BS FIR filters. For filter design problem, the MFO approach is able to achieve maximum stop-band attenuation, the lowest stop-band and pass-band ripples with adequate transition width. MFO algorithm performs better than a good number of existing methods, it has minimum stop band and pass band ripples with attenuation and maintaining a transition width which has the ability of obtaining better signal. It also minimum fitness value and transition width. Moreover, the experimental results have been compared with that of other high performing optimization algorithms such as NPSO, INPSO, GA, and PM. From the experimental results, PM produced some good results. Therefore, the outputs generated by the proposed approach are compared with that of PM exhaustively. Considering LP filter, the maximum stop-band attenuation, maximum stop-band ripple and transition width recorded for PM algorithm are $0.057154 \mathrm{~dB}, 0.0803$ and 4.5499 respectively, while, MFO is able to achieve superior parallel performance parameters for LP filter, i.e., 0.057326, 0.079 and 1.3682. MFO approach is able to enhance the maximum stop-band attenuation for HP, BP, BS filters when compared to results produced by PM algorithm. The MFO algorithm proved its dominance in two other performance parameters 'transition width' and 'fitness value' as compared to PM algorithm results for HP, BP, BS and FIR filters. Lastly, it can be concluded that the MFO approach is a promising algorithm with superior global optimum, robustness, computational efficiency compared to the other methods used in this work. Therefore, the objectives of this research have been achieved. The filter coefficient of the FIR filters has been obtained. The magnitude response (pass band, stop band ripples and fitness value) using the MFO algorithm has been determined and evaluated using standard performance metrics. And the performance of the proposed system has been compared with that of other existing algorithms.

This research is of great significance in today's world and future digital signal processing. Digital filters have 
found application in digital communication system, biomedical signal processing, electronics and computer music. The main advantages of Finite Impulse Response FIR filter include its ability to have an exact linear phase response, design and noise issues are less complex, it is stable, computational efficient realization exist for FIR filters realization are inherently stable and free of limit cycle oscillations when implemented on a finite-word length digital system. It is a filter with unity bandwidth and impedance which can be used for audio application, anti-imaging filter, anti-aliasing filter, receivers etc. Filters will be able to eliminate all unwanted signals or frequencies to have clearer signal. In the future, more experiments will be conducted using MFO algorithm to filter real signals like audio and images. Also, subsequent research will delve into using other existing optimization algorithm that are yet to be exploited by other researchers in the design and optimization of both FIR and IIR digital filters. These algorithms include Least Square Support Vector Machine (LS-SVM)[26], k-Nearest Neighbor [27], hybrid of decision tree and genetic algorithm [28], hybrid of Fuzzy logic and Convolution Neural Network (CNN) [29], and hybrid CNN and SVM model [30].

\section{Reference}

[1] R. Dastres, and M. Soori, “A Review in Advanced Digital Signal Processing Systems,” International Journal of Electrical and Computer Engineering; 2021.

[2] A. K. Dwivedi and R. N. Patel, "Digital filter design using quantum-inspired multiobjective cat swarm optimization algorithm," In Quantum inspired computational intelligence, Morgan Kaufmann, pp. 327-359; 2017.

[3] S. H. Mousavinezhad, "Computer Aided Design of Digital Filters". Michigan; 1998.

[4] A. K. Jatana and D. S. Sidhu, "Design of Digital FIR High Pass Filter Using Particle Swarm Optimization (PSO) Technique," International Journal of Scientific Research Engineering \& Technology (IJSRET), vol. 4, no. 5, pp. 472-479; 2015.

[5] S. Mondal, R. K. Vasundhara, D. Mandal and S. P. Ghoshal, "Linear phase high pass FIR filter design using improved particle swarm optimization," World academy of science, engineering and technology, vol. 60, pp. 1621-1627; 2011.

[6] G. Shilpi, R. Pooja, and R. K. Prasad, "Design and Optimization of Stepped Impedance Low Pass Filter using ADS Simulation tool at $5 \mathrm{GHz}$," International Journal of Advanced Research in Computer Engineering \& Technology IJARCET, vol. 3, no. 5, pp. $1813 ; 2014$.

[7] N. M. Shehu, A. S. Gidado, Y. S. Wudil, and U. A. Gora, "Performance Analysis of FIR Low Pass Filter Design using Blackman and Flat Top Window Methods," International Journal of Engineering Science and Computing, vol. 6, no. 4; 2016.

[8] I. B. Khalid, and A. M. A. Nemer, "Improved Particle Swarm Optimization for FIR filter design," Research gate, King Fahad University of Petroleum and Minerals, pp. 1-7; 2019.

[9] K. Kaur, U. Singh, and R. Salgotra, “An enhanced moth flame optimization," Neural Computing and Applications, vol. 32, no. 7, pp. 2315-2349; 2020.

[10] P. Vijay, and M. V. Sudhaka, "Comparison Of Various FIR Low Pass Filter Design Techniques With PSO Algorithm," International Journal of Advanced Research in Computer and Communication Engineering, vol. 1, no. 9; 2012.

[11] R. Thakur, and K. Khare, "High speed FPGA implementation of FIR filter for DSP applications," International Journal of Modeling and Optimization, vol. 3, no. 1, pp. 92-94; 2013.

[12] H. Kaur, and B. Dhaliwal, "Design of Low Pass FIR Filter Using Artificial Neural Network," International Journal of Information and Electronics Engineering, vol. 3, no. 2, pp. 204; 2013.

[13] C. Uthayakumar, and B. J. Rabi, "Design of FIR filter using window method," In IPASJ International Journal of Electronics and Communication. Karpagam University, coimbatore and Shri Andal Alagar college of Engineering, vol. 2, no. $10 ; 2014$.

[14] A. K. Maan, B. Singh, and D. S. Sidhu, "Design of High Order Digital IIR Filter using Heuristic Optimization Technique," International Journal of Advanced Research in Computer Science and Software Engineering, vol. 4, no. 10, pp. 1-7, 2014.

[15] N. Brar, B. Singh, and D. S. Sidhu, "Soft Computing Technique for the Design of Band Pass FIR Digital Filter," Soft Computing, vol. 5, no. 7; 2016.

[16] K. Neelam, and J. Priyanka, "Design of FIR Low Pass Filter using Particle Swarm Optimization Algorithm," International Journal of Advanced Research and Publications (IJARP), vol. 1, no. 3, pp. 31-35; 2017.

[17] S. Parampal and S. Balwinder. "Design of Digital FIR Filter using Hybrid SIMBO-GA Technique", International Journal of Hybrid Information Technology, vol. 8, no. 11, pp. 91-100; 2015.

[18] S. Kar, S. Das, and P. K. Ghosh, "Applications of neuro fuzzy systems: A brief review and future outline," Applied Soft Computing, vol. 15, pp. 243-259; 2014.

[19] P. A. Khaire, and N. V. Thakur, "Image edge detection based on soft computing approach," International Journal of Computer Applications, vol. 51, no. 8; 2012.

[20] R. Martinek, J, Vanus, P. Bilik, T. Stratil, and J. Zidek, “An efficient control method of shunt active power filter using adaline,” IFAC-PapersOnLine, vol. 49, no. 25, pp. 352-357; 2016.

[21] S. Dhabal, and P. Venkateswaran, "Two-dimensional IIR filter design using simulated annealing based particle swarm optimization. Journal of Optimization, 2014.

[22] R. Raja, T. S. Sinha, and R. P. Dubey, "Recognition of human-face from side-view using progressive switching pattern and soft-computing technique," Association for the advancement of modelling and simulation techniques in enterprises, Advance B, vol. 58, no. 1, pp. 14-34; 2015.

[23] M. Kumar, S. K. Mishra, and S. S. Sahu, "Cat swarm optimization based functional link artificial neural network filter for Gaussian noise removal from computed tomography images," Applied Computational Intelligence and Soft Computing, 2016.

[24] S. Lakra, T. V. Prasad, and G. Ramakrishna, "Speech Signal Filters based on Soft Computing Techniques: A Comparison," arXiv preprint arXiv: $1209.4445 ; 2012$.

[25] S. Mirjalili, "Moth-flame optimization algorithm: A novel nature-inspired heuristic paradigm," Knowledge-based systems, 89, 228-249; 2015. 
[26] Dattaprasad Torse, Veena Desai, Rajashri Khanai," Classification of EEG Signals in a Seizure Detection System Using Dual Tree Complex Wavelet Transform and Least Squares Support Vector Machine", International Journal of Image, Graphics and Signal Processing (IJIGSP), Vol.10, No.1, pp. 56-64, 2018.DOI: 10.5815/ijigsp.2018.01.07

[27] Shivanand S. Gornale, Pooja U. Patravali, Archana M. Uppin, Prakash S. Hiremath, "Study of Segmentation Techniques for Assessment of Osteoarthritis in Knee X-ray Images", International Journal of Image, Graphics and Signal Processing (IJIGSP), Vol.11, No.2, pp. 48-57, 2019.DOI: 10.5815/ijigsp.2019.02.06

[28] Pavan Sai Diwakar Nutheti, Narayan Hasyagar, Rajashree Shettar, Shankru Guggari, Umadevi V,"Ferrer diagram based partitioning technique to decision tree using genetic algorithm", International Journal of Mathematical Sciences and Computing (IJMSC), Vol.6, No.1, pp.25-32, 2020. DOI: 10.5815/ijmsc.2020.01.03

[29] Prashengit Dhar, Sunanda Guha, " Skin Lesion Detection Using Fuzzy Approach and Classification with CNN ", International Journal of Engineering and Manufacturing (IJEM), Vol.11, No.1, pp. 11-18, 2021. DOI: 10.5815/ijem.2021.01.02

[30] Sumit S. Lad, Amol C. Adamuthe, "Malware Classification with Improved Convolutional Neural Network Model", International Journal of Computer Network and Information Security (IJCNIS), Vol.12, No.6, pp.30-43, 2020. DOI: 10.5815/ijcnis.2020.06.03

\section{Authors' Profiles}

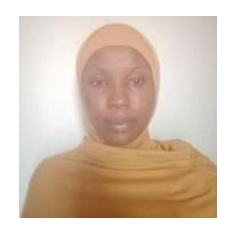

Zainab M. Adamu is a lecturer in the Department of Computer Engineering, University of Maiduguri. She receives her Bachelor of Engineering in Computer Engineering and Master of Engineering in Computer Engineering from University of Maiduguri, Nigeria. She is a member of Nigerian Society of Engineers (NSE) and Council for the Regulation of Engineering in Nigeria (COREN). Her research interest centres on microcontroller, softcomputing, signal processing, machine learning algorithms, and embedded systems. She has presented papers in conferences and published papers in some reputable journals.

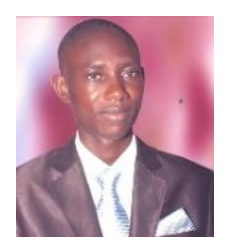

Emmanuel G. Dada is an Associate Professor of Computer Science at the at Department of Mathematical Sciences, University of Maiduguri, Nigeria. He received his $\mathrm{PhD}$ in Computer Science from Universiti Malaya, Malaysia (UM), MSc in Computer Science from University of Ibadan, Ibadan (UI), Nigeria and a Bachelor of Science in Computer Science from the University of Ilorin, Ilorin, Nigeria. His current research interests are in Softcomputing Techniques and Machine Learning Algorithms with their application to Medical Imaging, Cyber Security, COVID-19 detection, diagnosis, and Surveillance. He has published several over 70 academic papers in reputable International and local journals, conference proceedings and book chapters. He has been appointed as a reviewer of several ISI and Scopus indexed international journals such as ACM Survey, IEEE Access, Lecture Notes in Computational Vision and Biomechanics. He is a member of IEEE, International Society for Knowledge Organization (ISOK-WA), International Association of Engineers (IAENG) and Computer Professionals Registration Council of Nigeria (CPN). He is presently a lecturer at Department of Mathematical Sciences (Computer Science Unit), University of Maiduguri, Nigeria.

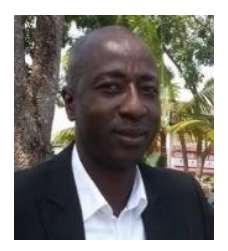

Stephen B. Joseph is a lecturer at the Department of Computer Engineering, University of Maiduguri, Nigeria. He received his Ph.D. degree in Electrical Engineering from the Universiti Teknologi Malaysia, in 2017, M.Eng. degree in Electrical \& Electronics Engineering (Electronics) from University of Maiduguri, Nigeria in 2012 and B.Tech degree in Computer Science \& Mathematics from Federal University of Technology Minna, Nigeria in 2000. He is currently a Lecturer with the Department of Computer Engineering, Faculty of Engineering, University of Maiduguri, Nigeria. His research interests are in Network algorithmic, Artificial Intelligence, optimization techniques and computer communication networks.

How to cite this paper: Zainab Muhammad Adamu, Emmanuel Gbenga Dada, Stephen Bassi Joseph, "Moth Flame Optimization Algorithm for Optimal FIR Filter Design", International Journal of Intelligent Systems and Applications(IJISA), Vol.13, No.5, pp.2434, 2021. DOI: $10.5815 /$ ijisa.2021.05.03 